\title{
Micronutrients and host resistance to viral infection
}

\author{
Melinda A. Beck ${ }^{1,2 *}$ and Colette C. Matthews ${ }^{2}$ \\ Departments of ${ }^{1}$ Pediatrics and ${ }^{2}$ Nutrition, CB \#7220, University of NC at Chapel Hill, Chapel Hill, NC 27599-7220, USA
}

\begin{abstract}
Previous work in our laboratory demonstrated that a virus could undergo rapid mutation in a host deficient in Se, leading to a normally avirulent virus acquiring virulence due to genome changes. Once these mutations occur, even a host with adequate Se-nutriture is susceptible to the newly virulent virus. What influence does the deficiency in Se have on the immune response of the host? Infection with myocarditic strains of coxsackievirus induces an inflammatory response in the cardiac tissue. It is this immune response that induces the heart damage, rather than direct viral effects on the heart tissue. Chemokines are chemo-attractant molecules that are secreted during an infection in order to attract immune cells to the site of the injury, and have been found to be important for the development of coxsackievirus-induced myocarditis. We found that a deficiency in Se influences the expression of mRNA for the chemokine monocyte chemo-attractant protein-1, which may have implications for the development of myocarditis in the Se-deficient host. Expression of mRNA for interferon- $\gamma$ was also greatly decreased in the Se-deficient animal. Thus, a deficiency in Se can have profound effects on the host as well as on the virus itself. How the alteration of the immune response of the Se-deficient animal affects the development of the virulent genotype remains to be answered.
\end{abstract}

Selenium: Coxsackievirus-induced myocarditis: Immune response: Chemokines

The influence of micronutrients on the ability of the host to respond to a viral infection has been studied for a number of years (Scrimshaw et al. 1968; Scrimshaw, 1975; Bendich \& Chandra, 1990; Good \& Lorenz, 1992). Recently, our laboratory has demonstrated that the virus itself may respond to the nutritional status of the host (Beck et al. 1995). An avirulent strain of coxsackievirus (CV) has been shown to develop virulence, due to mutations in the viral genome, as a consequence of replicating in a Se-deficient host. Once these mutations occur, even mice with normal Se status will develop disease when infected with the newly virulent virus.

The driving force behind the change in virulence is thought to be due to increased oxidative stress of the host, due to a lack of antioxidant protection. Se is an essential component of the antioxidant enzyme glutathione peroxidase. What effect does the increased oxidative stress status of the host have on the immune response of the deficient animals? How does the increase in oxidative stress contribute to the immunopathology of the disease? Are there viruses other than $\mathrm{CV}$ that may be susceptible to increased oxidative stress in the host? The answer to these questions has important implications for the ability of the host to resist viral infection, as well as the ability of the virus to adapt to changing host conditions.

\section{Brief summary of earlier coxsackievirus B3 work}

Our work with CVB3 virus developed as a consequence of studying Keshan disease (KD). KD, first reported in China in the 1930s, is a cardiomyopathy that affects mainly women and children ( $\mathrm{Gu}, 1983)$. The disease is associated with a deficiency in Se, and can be prevented with $\mathrm{Se}$ supplementation (Keshan Disease Research Group of the Chinese Academy of Medical Sciences, 1979). However, because of the seasonal and annual incidence of the disease, an infectious cofactor was also suspected as being necessary for the development of KD. Since enteroviruses, and particularly $\mathrm{CV}$, are known to infect the heart, scientists in China examined blood and tissue samples of KD patients for the presence of these viruses. They were successful in isolating $\mathrm{CV}$ from the blood and tissue of a number of $\mathrm{KD}$ patients ( $\mathrm{Su}$ et al. 1979). More recently, the technique of reverse transcriptase-polymerase chain reaction has been utilized to identify $\mathrm{CV}$ sequences in archived tissue from $\mathrm{KD}$ victims ( $\mathrm{Li}$ et al. 1995). All these results suggest a

\footnotetext{
Abbreviations: CV, coxsackievirus; IFN- $\gamma$, interferon- $\gamma$; KD, Keshan disease; MCP-1, monocyte chemo-attractant protein-1; MIP-1, macrophage inflammatory protein-1; RANTES, regulated on activation, normal T-cell expressed and secreted.

*Corresponding author: Professor Melinda Beck, fax +1919966 0135, email melinda_beck@unc.edu
} 
relationship between Se deficiency, infection with CV and the development of KD.

In order to investigate further the relationship between $\mathrm{KD}$ and $\mathrm{CV}$ infection, we utilized a murine model of CVB3-induced myocarditis. Mice were fed a diet either adequate or deficient in Se for 4 weeks, and then infected with a normally benign strain of coxsackievirus B3 (CVB3/0). Under adequate dietary conditions, this virus strain will replicate in heart tissue, but will not cause myocarditis. However, the Se-deficient mice developed moderate myocarditis after infection with CVB3/0, whereas the Se-adequate mice did not (Beck et al. 1994). The virus titre was higher in the Se-deficient animals, suggesting an impairment of the immune defence against the virus. Indeed, we found that $\mathrm{T}$-cell proliferative responses against both mitogen and specific viral antigen were markedly decreased in the infected Se-deficient mice. Interestingly, the ability of the Se-deficient mice to mount a neutralizing antibody response was not affected by the Se deficiency.

It has been shown by many studies that a nutritional deficiency will inhibit the immune response (Bendich \& Chandra, 1990; Good \& Lorenz, 1992). Decreased antibody responses, impaired macrophage function and increased susceptibility to infections have all been reported as a consequence of Se deficiency (Reffet et al. 1988a,b). Thus, our results were similar to those reported previously by other researchers. In addition to looking at the host response, we reasoned that the virus itself might also be affected as a consequence of replicating in a Se-deficient host. In order to test this possibility we passed virus that was isolated from Se-deficient mice back into Se-adequate mice. If the virulence of the virus in the Se-deficient mice was due entirely to host factors, then the Se-adequate mice infected with virus from the Se-deficient animals should not develop myocarditis. However, the opposite occurred. The Seadequate mice developed moderate myocarditis. Se-adequate mice infected with virus isolated from Se-adequate mice did not develop any myocarditis, demonstrating that viral passage alone did not alter the virus.

This surprising result strongly suggested that a change in the viral genome occurred as a consequence of replicating in a Se-deficient host. Indeed, sequencing confirmed that mutations did occur in the viral genome (Beck et al. 1995). The virus that replicated in the Se-deficient host had six mutations in the viral genome, all of which are associated with a virulent phenotype. No changes in the viral genome occurred in virus that had replicated in the Se-adequate host. Our results demonstrated for the first time that the nutritional status of the host could exert a profound effect on a virus, changing the genome of an avirulent virus to a virulent one.

\section{Immune response of the selenium-deficient mice infected with a normally benign strain of coxsackievirus B3}

Inflammation is the hallmark of CVB3-induced myocarditis (Woodruff, 1980). In the mouse the development of inflammation occurs within $6 \mathrm{~d}$ after infection with a myocarditic strain of CVB3. Following an intraperitoneal injection the virus replicates during the first several days in the liver, next travelling to the heart where an additional round of replication takes place. Once in the heart the virus induces a brisk immune response, characterized by the appearance of macrophages and natural killer cells early, then later replaced with a T-cell infiltrate. Woodruff \& Woodruff (1974) demonstrated the importance of the immune infiltrate to the disease process. Using antibodies to deplete mice of T-cells they found that an infection with a virulent strain of CVB3 did not induce any myocarditis. Additional studies using nude mice also confirmed this result; nude mice were protected from CVB3-induced myocarditis. These results highlight the idea that the inflammatory response to CVB3 induces the myocarditis, rather than virus replication in and of itself (O'Connell \& Robinson, 1985; Leslie, 1989). Indeed, the amyocarditic strain of CVB3 (CVB3/0) does not induce an inflammatory response, even though the virus replicates in and can be recovered from the heart tissue of infected animals.

Why do the immune cells traffic to the heart after infection with a myocarditic strain of CVB3? One possibility is the elaboration of chemokines, chemo-attractant molecules produced by immune cells as well as other types of cells (Zlotnik \& Yoshie, 2000). The importance of the chemokine response to development of myocarditis was shown with the use of 'knockout' mice. Macrophage inflammatory protein-1 (MIP-1) $\alpha$ is a chemokine that is chemotactic for activated CD8+ T-cells and natural killer cells. In vitro, MIP- $1 \alpha$ has many of the same functions as MIP-1 $\beta$ and RANTES (regulated on activation, normal T-cell expressed and secreted).

Cook et al. (1995) demonstrated that MIP- $1 \alpha$ 'knockout' mice infected with a virulent strain of CVB3 (CVB3/20) did not develop any myocarditis, whereas the wild-type mice developed moderate myocarditis when infected with the myocarditic strain of CVB3. Thus, a lack of MIP- $1 \alpha$ could completely protect the mice from developing myocarditis. Virus was recovered from the hearts of both 'knockout' and wild-type mice, demonstrating that the lack of MIP- $1 \alpha$ did not affect the ability of the virus to infect and replicate in heart tissue. These results demonstrate that the production of MIP- $1 \alpha$ is essential for the development of CVB3-induced myocarditis.

In order to determine the role of MIP- $1 \alpha$ as well as that of other chemokines in the development of myocarditis in Se-deficient animals, we used an RNAse protection assay to look at expression of mRNA for these chemo-attractants in the heart. At weaning mice were fed Se-deficient (no added $\mathrm{Se}$ in a diet with undetectable Se levels) or Se-adequate $\left(0 \cdot 2 \mu \mathrm{g}\right.$ as $\left.\mathrm{Na}_{2} \mathrm{SeO}_{3} / \mathrm{g}\right)$ diets for 4 weeks. After 4 weeks of feeding, mice were infected with CVB3/0, the amyocarditic strain of CVB3. At various times after infection mice were killed and the hearts removed for study. Total RNA was isolated from the hearts and used in a Riboquant RNAse protection assay (BD PharMingen, San Diego, CA, USA).

As shown in Fig. 1, mRNA expression of RANTES, eotaxin, MIP- $1 \alpha$ and MIP- $1 \beta$ were not statistically different between CVB3/0-infected Se-adequate and Se-deficient groups. RANTES, MIP-1 $\beta$ and eotaxin peaked between days 7 and 10 post-infection and MIP- $1 \alpha$ expression was highest at day 10 post-infection, the last day of measurement. 

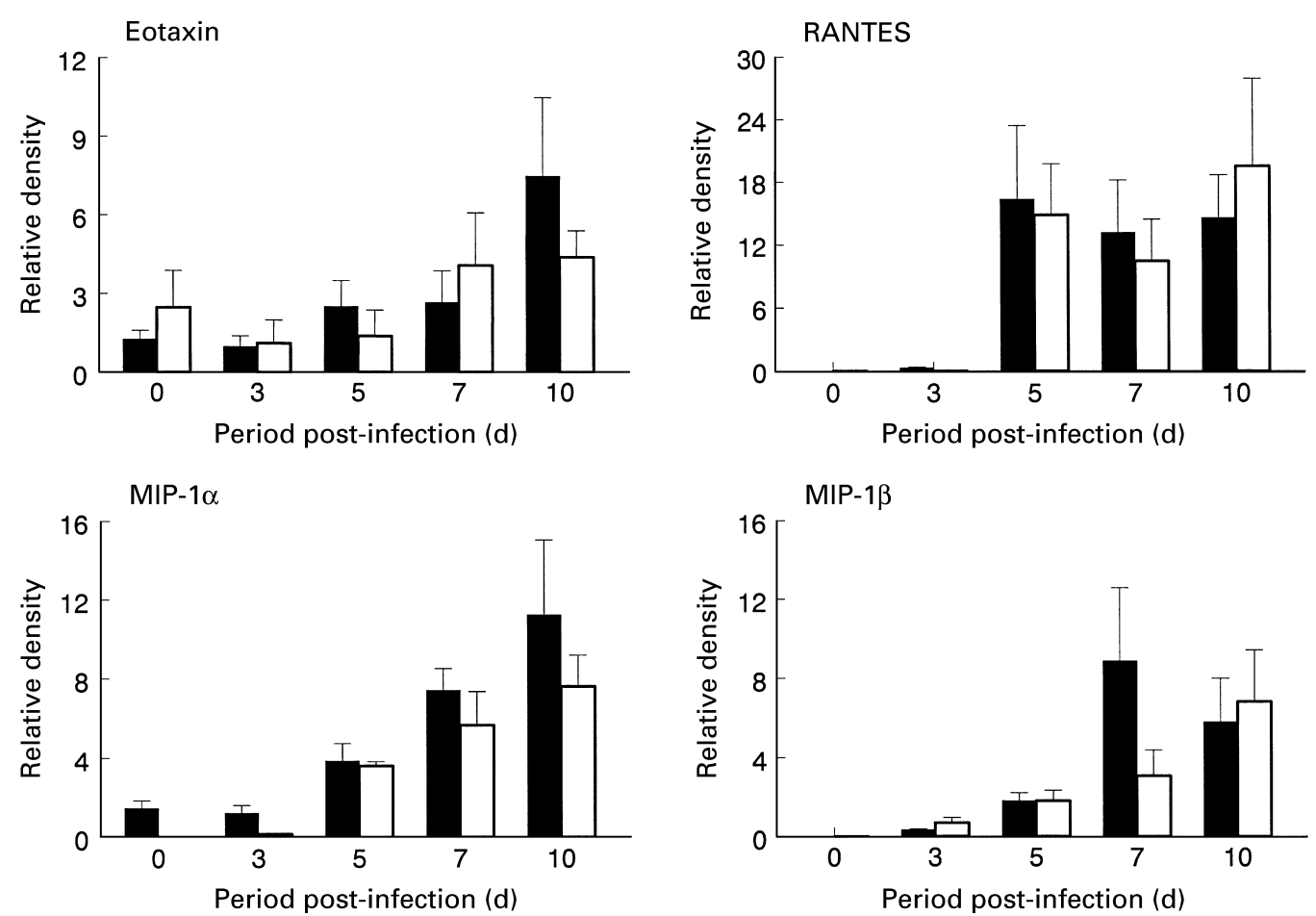

Fig. 1. Heart mRNA levels for chemokines from selenium-adequate $(\square)$ and selenium-deficient $(\square)$ mice. Total RNA was isolated from both groups using TRIzol reagent (GIBCOBRL Life Technologies, Grand Island, NY, USA) and quantified at $260 \mathrm{~nm}$. Total RNA $(10 \mu \mathrm{g})$ was used in the RiboQuant Multiprobe Protection Assay (BD PharMingen, San Diego, CA, USA). For quantification, gels were scanned and bands analysed densitometrically using ImageGauge Software (Fuji Films/Fuji Medical Systems, Bridgeport, CT, USA). For details of dietary regimen and experimental procedures, see p. 582. Values are means with their standard errors represented by vertical bars for five animals. There were no statistical differences between groups. MIP-1, macrophage inflammatory protein-1; RANTES, regulated on activation, normal T-cells expressed and secreted.

Interestingly, expression of mRNA for monocyte chemoattractant protein-1 (MCP-1) was significantly $(P<0 \cdot 05)$ different between hearts of Se-deficient $v$. Se-adequate animals (Fig. 2). mRNA levels for MCP-1 peaked at day 7 for the Se-adequate mice, then dropped off at day 10. However, the MCP-1 mRNA level for Se-deficient mice was low at day 7, but showed a dramatic increase at day 10 post-infection.

These results suggest that MCP-1 may play a role in the development of myocarditis. MCP-1 is also involved in the trafficking of T-cells. Although previous work (Cook et al. 1995) had demonstrated an importance for MIP- $1 \alpha$ in nutritionally adequate mice, we did not find a difference between Se-adequate and Se-deficient mice with respect to expression of the mRNA for this chemokine. There could be several reasons for this result. First, only mRNA levels were measured. We did not look at protein levels for these chemokines. It is possible that a deficient diet may interfere with translation of the mRNA into protein. Investigation into the secretion of these proteins post-infection is ongoing in order to determine if mRNA translation is inhibited in Se-deficient animals. Second, the immune response of a MIP- $1 \alpha$ 'knockout' animal may be altered in areas other than a lack of MIP-1 $\alpha$. The ability of the 'knockout' animal to respond to viral infection may be very different from that

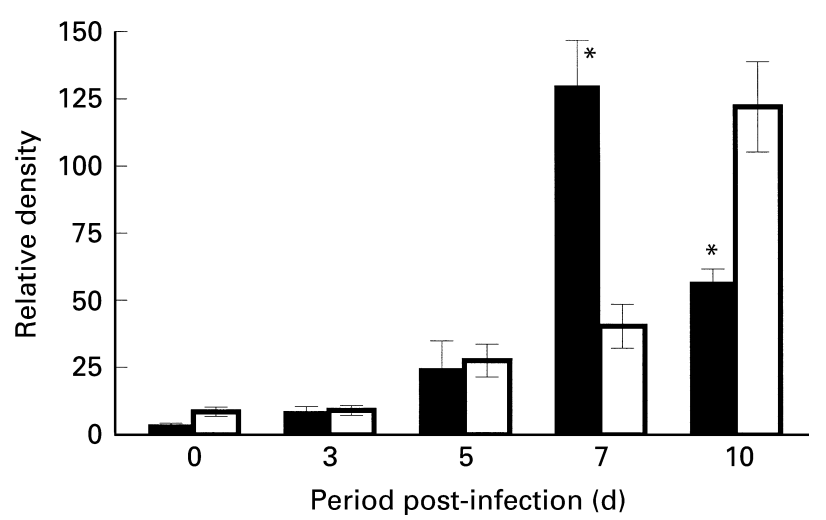

Fig. 2. Heart mRNA level for MCP-1 from selenium-adequate ( $\square$ ) and selenium-deficient $(\boldsymbol{\square})$ mice. Total RNA was isolated from both groups using TRIzol reagent (GIBCOBRL Life Technologies, Grand Island, NY, USA) and quantified at $260 \mathrm{~nm}$. Total RNA $(10 \mu \mathrm{g})$ was used in the RiboQuant Multiprobe Protection Assay (BD PharMingen, San Diego, CA, USA). For quantification, gels were scanned and bands analysed densitometrically using Image Gauge Software (Fuji Films/Fuji Medical Systems, Bridgeport, CT, USA). For details of dietary regimen and experimental procedures, see $\mathrm{p}$. 582. Values are means with their standard errors represented by vertical bars for five animals. Mean values were significantly different from those for the selenium-adequate group: ${ }^{*} P<0.05$. 
of a Se-deficient animal. Multiple pathways may be important for the development of myocarditis, not all of which involve MIP- $1 \alpha$. Thus, when MIP- $1 \alpha$ is present, as in the Se-deficient animals, other chemokines may be more important for the infection process, such as MCP-1. Further studies are necessary to understand this complex interplay of chemokines and the development of disease.

A second interesting immune finding concerns interferon- $\gamma($ IFN- $\gamma)$. As shown in Fig. 3, mRNA expression for IFN- $\gamma$ was weakly expressed in the Se-deficient mice, whereas strong expression of IFN- $\gamma$ occurred in the Se-adequate mice, peaking at day 7 post-infection. IFN- $\gamma$ has been shown to be an important anti-viral cytokine, and is also a powerful activator of macrophages. Our previous studies have demonstrated that viral titres are elevated in the CVB3/0-infected Se-deficient mice, which suggests that a lack of IFN- $\gamma$ in these animals may contribute to the higher titre. Expression of mRNA for other cytokines, including interleukins 1, 2, 4, 5, 6 and 15, was not statistically different between Se-deficient and Se-adequate animals.

\section{Conclusion}

Our previous studies have demonstrated that infection of Se-deficient mice with an avirulent CVB3 strain results in conversion of the avirulent strain into a virulent one. Since the chemokine response has been shown to be important for the generation of an inflammatory response, we examined both chemokine and cytokine responses post-infection. The alteration in the expression of mRNA for MCP-1 in the Se-deficient mice as compared with the Se-adequate mice may contribute to the pathogenicity. Of particular interest is a significant lack of IFN- $\gamma$ mRNA production in the Sedeficient animals. In view of its potent anti-viral effects, a lack of IFN- $\gamma$ may contribute directly to increased viral virulence by allowing the virus to replicate to a higher titre, thus enhancing the possibility for viral mutations to occur. Recently, we (Nelson et al. 2000) have begun looking at the effects of Se deficiency on influenza virus infection, and preliminary results suggest that a deficiency in Se can alter the pathogenesis of influenza-induced pneumonitis in Se-deficient mice. Further investigation of the effects of a Se deficiency on virus-induced pathogenicity remains to be explored.

\section{References}

Beck MA, Kolbeck PC, Rohr LH, Shi Q, Morris VC \& Levander OA (1994) Amyocarditic coxsackievirus becomes myocarditic in selenium deficient mice. Journal of Medical Virology 43, 166-170.

Beck MA, Shi Q, Morris VC \& Levander OA (1995) Rapid genomic evolution of a non-virulent Coxsackievirus B3 in selenium-deficient mice results in selection of identical virulent isolates. Nature Medicine 1, 433-436.

Bendich A \& Chandra RK (1990) Micronutrients and immune functions. Annals of the New York Academy of Sciences 587, $168-180$.

Cook DN, Beck MA, Coffman T, Kirby SL, Sheridan JF, Pragnell IB \& Smithies O (1995) Requirement of MIP-1 $\alpha$ for inflammatory response to viral infection. Science 269, $1583-1585$.

Good RA \& Lorenz E (1992) Nutrition and cellular immunity. International Journal of Immunopharmacology 14, 361-366.

Gu BQ (1983) Pathology of Keshan disease. A comprehensive review. Chinese Medical Journal 96, 251-261.

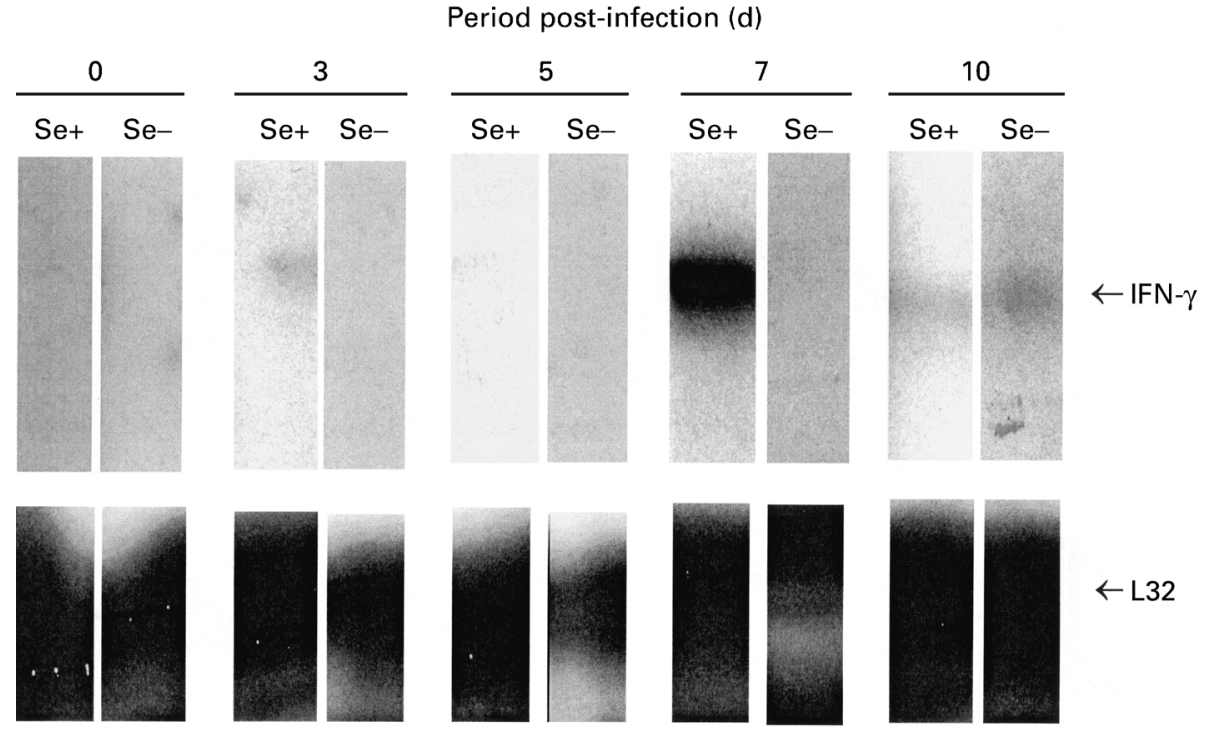

Fig. 3. Heart mRNA level for interferon- $\gamma(\mathrm{IFN}-\gamma)$ for selenium-adequate (Se+) and seleniumdeficient (Se-) mice. Total RNA was isolated from both groups using TRIzol reagent (GIBCOBRL Life Technologies, Grand Island, NY, USA) and quantified at $260 \mathrm{~nm}$. Total RNA (10 $\mu \mathrm{g})$ was used in the RiboQuant Multiprobe Protection Assay (BD PharMingen, San Diego, CA, USA). For quantification, gels were scanned and bands analysed densitometrically using Image Gauge Software (Fuji Films/Fuji Medical Systems, Bridgeport, CT, USA). L32 is a housekeeping gene used to demonstrate total amount of mRNA loaded on the gel. Each gel is representative of five animals. For details of dietary regimen and experimental procedures, see p. 582. 
Keshan Disease Research Group of the Chinese Academy of Medical Sciences (1979) Observations on effect of sodium selenite in prevention of Keshan disease. Chinese Medical Journal 92, 471-476.

Leslie K (1989) Clinical and experimental aspects of viral myocarditis. Clinical Microbiology Reviews 2, 191-197.

Li Y, Yang Y \& Chen H (1995) Detection of enteroviral RNA in paraffin-embedded myocardial tissue from patients with Keshan disease by nested PCR. Chung Hua I Hsueh Tsa Chih 75, 344-345.

Nelson HK, Van Dael P, Schiffrin EJ, Brussow H, Blum S, Barclay D \& Beck MA (2000) Selenium deficiency enhances the pathogenicity of influenza virus infection. FASEB Journal 14, A536.

O'Connell J \& Robinson J (1985) Coxsackie viral myocarditis. Postgraduate Medical Journal 61, 1127-1131.

Reffett JK, Spears JW \& Brown TT Jr (1988a) Effect of dietary selenium and vitamin $\mathrm{E}$ on the primary and secondary immune response in lambs challenged with parainfluenza 3 virus. Journal of Animal Sciences 66, 1520-1528.
Reffett JK, Spears JW \& Brown TT Jr (1988b) Effect of dietary selenium on the primary and secondary immune response in calves challenged with infectious bovine rhinotracheitis. Journal of Nutrition 118, 229-235.

Scrimshaw NS (1975) Nutrition and infection. Progress in Food and Nutrition Science 1, 393-420.

Scrimshaw NS, Taylor CE \& Gordon JE (1968) Interactions of Nutrition and Infection. WHO Monograph Series no. 57, Geneva: WHO

Su C, Gong C, Li J, Chen L, Zhou D \& Jin Q (1979) Preliminary results of viral etiology of Keshan disease. Chinese Medical Journal 59, 466-472.

Woodruff JF (1980) Viral myocarditis. A review. American Journal of Pathology 101, 427-482.

Woodruff JF \& Woodruff JJ (1974) Involvement of T lymphocytes in the pathogenesis of coxsackie virus B3 heart disease. Journal of Immunology 113, 17-26.

Zlotnik A \& Yoshie O (2000) Chemokines: A new classification system and their role in immunity. Immunity 12, 121-127. 